\title{
The Effect of the Earth's Atmosphere on LSST Photometry
}

\author{
Alexandra S. Rahlin \\ Office of Science, Science Undergraduate Laboratory Internship (SULI) \\ Massachusetts Institute of Technology \\ Stanford Linear Accelerator Center \\ Stanford, CA
}

August 25, 2006

Prepared in partial fulfillment of the requirements of the Office of Science, Department of Energy's Science Undergraduate Laboratory Internship under the direction of David L. Burke at the Kavli Institute for Particle Astrophysics and Cosmology, Stanford Linear Accelerator Center.

Participant:

Signature

Research Advisor:

Signature 


\section{TABLE OF CONTENTS}

$\begin{array}{ll}\text { Abstract } & \text { ii }\end{array}$

$\begin{array}{ll}\text { Introduction } & 1\end{array}$

$\begin{array}{ll}\text { Materials and Methods } & 3\end{array}$

$\begin{array}{lr}\text { Results } & 6\end{array}$

$\begin{array}{ll}\text { Discussion and Conclusions } & 7\end{array}$

$\begin{array}{lr}\text { Acknowledgments } & 9\end{array}$

$\begin{array}{lr}\text { References } & 9\end{array}$ 


\begin{abstract}
The Effect of the Earth's Atmosphere on LSST Photometry. ALEXANDRA S. RAHLIN (Massachusetts Institute of Technology, Cambridge, MA 02139) DAVID L. BURKE (Kavli Institute for Particle Astrophysics and Cosmology, Stanford Linear Accelerator Center, Stanford, CA 94025).
\end{abstract}

The Large Synoptic Survey Telescope (LSST), a ground-based telescope currently under development, will allow a thorough study of dark energy by measuring, more completely and accurately than previously, the rate of expansion of the universe and the large-scale structure of the matter in it. The telescope utilizes a broadband photometric system of six wavelength bands to measure the redshifts of distant objects. The earth's atmosphere makes it difficult to acquire accurate data, since some of the light passing through the atmosphere is scattered or absorbed due to Rayleigh scattering, molecular absorption, and aerosol scattering. Changes in the atmospheric extinction distribution due to each of these three processes were simulated by altering the parameters of a sample atmospheric distribution. Spectral energy distributions of standard stars were used to simulate data acquired by the telescope. The effects of changes in the atmospheric parameters on the photon flux measurements through each wavelength band were observed in order to determine which atmospheric conditions must be monitored most closely to achieve the desired $1 \%$ uncertainty on flux values. It was found that changes in the Rayleigh scattering parameter produced the most significant variations in the data; therefore, the molecular volume density (pressure) must be measured with at most $8 \%$ uncertainty. The molecular absorption parameters produced less significant variations and could be measured with at most $62 \%$ uncertainty. The aerosol scattering parameters produced almost negligible variations in the data and could be measured with $>100 \%$ uncertainty. These atmospheric effects were found to be almost independent of the redshift of the light source. The results of this study will aid the design of the atmospheric monitoring systems for the LSST. 


\section{INTRODUCTION}

The Large Synoptic Survey Telescope (LSST) is a ground-based telescope that is currently under development to be stationed in Cerro Pachón, Chile. It is designed with a $3.5^{\circ}$ field of view and will take 3-gigapixel charge-coupled device (CCD) images of about 20,000 square degrees of the visible sky every three nights. The short time scale between consecutive images allows for time-lapse images of rapidly developing celestial objects such as supernovae. Additionally, the large amount of data to be collected by the telescope in very short periods of time will open the door for studying dark energy much more accurately than in the past.

The LSST will use a broadband photometric system, shown in Figure 1, to collect spectra over six wavelength bands between 350 and $1050 \mathrm{~nm}$. The photometric efficiencies as a function of wavelength for each of the LSST's six filter bands (U, G, R, I, Z and Y), calculated in [1], include each of the filter response functions as well as the quantum efficiency of the CCD camera (Figure 2).

The LSST will be used to make accurate measurements of redshifts in light coming from various distant celestial objects. The redshift $z$ with a measured wavelength $\lambda$ and an expected wavelength $\lambda_{0}$ is defined as the fractional difference between the two wavelengths:

$$
z=\frac{\lambda-\lambda_{0}}{\lambda_{0}}
$$

The redshift of an object can be measured by observing characteristic shapes in its spectral energy distribution (SED), such as in the Balmer absorption lines of hydrogen that are visible in the 400-500nm range. For an object moving away from the earth when light is emitted from it, the wavelength of each of its Balmer lines will appear closer to the red end of the spectrum than it would be if the object were at rest.

Another method of measuring redshifts is to take advantage of the LSST's broadband photometric system, which measures the total photon flux through each wavelength band. Certain redshift values correspond to characteristic flux ratios between the bands. Either 
type of redshift measurement depends on correctly analyzed SEDs, so the uncertainty in flux measurements must be minimized in order to obtain accurate results.

Measuring the redshifts of many objects accurately is critical for characterizing the nature of dark energy. The invariant line element $d s^{2}$ for a flat universe is defined as:

$$
d s^{2}=-c^{2} d t^{2}+a^{2}(t) d \vec{x}^{2}
$$

Edwin Hubble's observations of galactic redshifts [2] demonstrate that the universe is expanding, so the scale factor $a(t)$ is larger today than it was in the past. The rate of change of the scale factor is defined by the Hubble parameter:

$$
H(t)=\left(\frac{\dot{a}}{a}\right)^{2}
$$

This parameter determines whether the expansion is accelerating or decelerating. The scale factor can be measured over time, relative to the scale factor today $(t=0)$, as a function of the redshift $z$ of an object whose light began its travel to Earth at an earlier time $t$ :

$$
1+z=\frac{a(0)}{a(t)}
$$

Measuring the redshifts of many distant galaxies provides a large data sample for characterizing the time evolution of (3). Earlier results $[3,4]$ show that the expansion of the universe is accelerating; the cause of this acceleration is unknown and is attributed to "dark energy" - a form of energy that is not yet understood.

The Earth's atmosphere presents an obstacle in acquiring accurate redshift data, because the LSST is a ground-based telescope. As light enters the atmosphere from various objects in the sky, the spectrum acquired changes by various conditions such as fluctuations in air pressure, temperature, and particle content. This process is called atmospheric extinction. The transmittance of the light coming through the atmosphere at various wavelengths has a char- 
acteristic shape, shown in Figure 3. The shape of the distribution at shorter wavelengths is dominated by elastic Rayleigh scattering by air molecules much smaller than the wavelength of incident light. The intensity of Rayleigh scattering varies as $\lambda^{-4}$, so shorter wavelengths scatter more significantly. At longer wavelengths, the dominant effects are optical scattering by aerosols (dust, water droplets, silicon, etc.) with an approximate $\lambda^{-1}$ dependence, and molecular absorption of vapors $\left(\mathrm{H}_{2} \mathrm{O}, \mathrm{O}_{2}, \mathrm{O}_{3}\right.$, etc.) at wavelengths of 700-1000 nm.

Because the atmosphere above the telescope is changing constantly, it is difficult to isolate the spectral energy distribution (SED) of a distant star or galaxy due to changes created by the atmosphere. The goal of this project was to determine the stability of various known SEDs with respect to changes in atmospheric conditions. It was expected that most atmospheric fluctuations would affect the signal by only a few percent; however, it was found that the data were more sensitive to changes in the Rayleigh scattering parameter than to changes in the molecular absorption and aerosol scattering parameters. This has study determined which atmospheric conditions would need be monitored most closely in order to ensure that redshift data could be accurately corrected.

\section{MATERIALS AND METHODS}

\section{Generation of Atmospheric Extinction Data}

Atmospheric extinction distributions over wavelengths of 300 to $1200 \mathrm{~nm}$ were generated by making changes to the atmospheric distribution in Figure 3, which can be characterized by an exponential function with four free parameters:

$$
A\left(\lambda ; \alpha_{R}, \alpha_{M}, \alpha_{A}, \alpha_{P}\right)=\exp \left(-\left[k_{R}\left(\lambda ; \alpha_{R}\right)+k_{M}\left(\lambda ; \alpha_{M}\right)+k_{A}\left(\lambda ; \alpha_{A}, \alpha_{P}\right)\right]\right) .
$$


Each $k$ is called an extinction coefficient. The Rayleigh scattering extinction coefficient:

$$
k_{R}\left(\lambda ; \alpha_{R}\right)=\alpha_{R}\left(\frac{\lambda}{300}\right)^{-4.05}
$$

is driven mostly by the atmospheric pressure. The wavelength in (6) is normalized so that $\alpha_{R}$ is a dimensionless constant.

The molecular absorption extinction coefficient:

$$
k_{M}\left(\lambda ; \alpha_{M}\right)=\alpha_{M} f(\lambda)
$$

contains information about the shapes of the lines due to absorption by $\mathrm{H}_{2} \mathrm{O}, \mathrm{O}_{2}$, ozone and other airborne molecules. The water content of the atmosphere changes with humidity, so the molecular absorption parameter is affected by both temperature and pressure; however, for the purposes of this study, the functional form of $f(\lambda)$ was defined by the shape of the absorption lines in the sample distribution.

The aerosol scattering extinction coefficient:

$$
k_{A}\left(\lambda ; \alpha_{A}, \alpha_{P}\right)=\alpha_{A}\left(\frac{\lambda}{300}\right)^{-\alpha_{P}}, \quad \text { where } \quad 0.5<\alpha_{P}<1.5
$$

is affected by atmospheric temperature and pressure, as well as winds that spread aerosols throughout the atmosphere. The wavelength in (8) is again normalized so that $\alpha_{A}$ is dimensionless. The spectral index $\alpha_{P}$ varies with the size of the aerosols. The cross section for scattering by larger aerosols is less dependent on the wavelength of incident light, therefore, the spectral index is closer to zero.

The Rayleigh and aerosol scattering parameters $\alpha_{R, 0}$ and $\alpha_{A, 0}$ for the atmospheric distribution in Figure 3 (solid black line) were obtained from a nonlinear least-squares fit (dashed red line) to the function $\exp \left[-\left[k_{R}\left(\lambda ; \alpha_{R, 0}\right)+k_{A}\left(\lambda ; \alpha_{A, 0}, 1\right)\right]\right]$. The result of the fit determined the initial values of $\alpha_{R, 0}=1.089$, and $\alpha_{A, 0}=0.071$ with a $\chi^{2}$ per degree of freedom of 5.74 . 
The large $\chi^{2}$ value is due mostly to the fact that the fit does not explicitly include the ozone absorption dip (near 600nm in Figure 3). Parameters $\alpha_{M, 0}$ and $\alpha_{P, 0}$ were both initialized to 1. New atmospheric distributions were generated by varying each initial $\alpha$ parameter by factors of up to $\pm 100 \%$. For example, changing $\alpha_{R}$ to $\alpha_{R}=0.1 \alpha_{R, 0}$ decreased the Rayleigh scattering extinction coefficient by a factor of ten, thus generating a new atmospheric extinction distribution, $A\left(\lambda ; 0.1 \alpha_{R, 0}, \alpha_{M, 0}, \alpha_{A, 0}, \alpha_{P, 0}\right)$.

Various regions of the $\left(\alpha_{R}, \alpha_{M}, \alpha_{A}\right)$ parameter space were explored by fixing two of these three factors at their initial values and varying the third by up to $\pm 100 \%$. The ranges of atmospheric extinction distributions generated by this method are shown in Figure 4 . The effects of each of these distributions were quantified as discussed in the following section. The $\left(\alpha_{A}, \alpha_{P}\right)$ parameter space was explored as well.

\section{Measurement of Atmospheric Extinction}

Several sets of standard data were used to analyze the atmospheric effects on LSST photometry. Spectral energy distributions (SEDs) for various stars were collected from [5]. The SED of a giant star from the catalog is shown in Figure 5.

The SED data $S(\lambda)$ were integrated with the response $T(\lambda ; f)$ of each filter band $f$ to simulate the true photon flux $\Phi_{\text {true }}(f, S)$ through each filter band:

$$
\Phi_{\text {true }}(f, S)=\int S(\lambda) T(\lambda ; f) d \lambda
$$

The measured photon flux $\Phi_{\text {meas }}(\lambda ; f, S, A)$ of each star was simulated by integrating its SED with an atmospheric extinction distribution $A\left(\lambda ; \alpha_{R}, \alpha_{M}, \alpha_{A}, \alpha_{P}\right)$ and the response of a particular LSST filter $T_{L S S T}(\lambda ; f)$ that included the CCD quantum efficiency:

$$
\Phi_{\text {meas }}(f, S, A)=\int S(\lambda) A\left(\lambda ; \alpha_{R}, \alpha_{M}, \alpha_{A}, \alpha_{P}\right) T_{L S S T}(\lambda ; f) d \lambda
$$

The filter response functions are shown in Figure 2, and Figure 5 displays the true and 
measured flux per wavelength through each filter band for a sample giant star.

A correction factor $C$ for approximating the true flux from measured values was calculated for each filter band from the subset of Dwarf stars in the star catalog:

$$
C(f, A)=\frac{1}{N} \sum_{\text {Dwarfs }} \frac{\Phi_{\text {true }}(f, S)}{\Phi_{\text {meas }}(f, S, A)}
$$

Corrected flux values, $\Phi_{\text {corr }}(f, S, A)=C(f, A) \Phi_{\text {meas }}(f, S, A)$, were obtained for each of the giant and supergiant stars in [5] and plotted against their true values. Trends in the $\Phi_{\text {corr }}$ distributions were measured as functions of variations $(\Delta \alpha)$ in each $\alpha$ parameter; i.e. a distribution generated using $\alpha_{R}=\alpha_{R, 0}$ corresponded to $\Delta \alpha_{R}=0 \%$. Figure 6 shows the $\Phi_{\text {corr }}$ distribution in the G band with a third-order polynomial fit, along with the ideal line $\left(\Phi_{c o r r}=\Phi_{t r u e}\right)$. Corrections to the measured flux were expected to fall within $1-2 \%$ of the true flux for each SED and filter. For each filter band, trends in the data generated from the variations on the reference atmosphere were observed by means of changes in the mean bias of the fit from the ideal line, and the width of the distribution about the ideal line.

\section{RESULTS}

Figure 7 shows the residuals of each corrected flux distribution from the ideal line in the $G$ band for various values of $\Delta \alpha_{R}$, with the remaining three atmospheric parameters fixed to their initial values. $\Delta \alpha_{R}$ was varied at uniform intervals between $\pm 20 \%$, while the remaining three parameters were varied between $-80 \%$ and $+100 \%$.

The trends in the bias of each distribution from the ideal line and the width about the ideal line are shown in Figure 8 for the $\mathrm{G}$ band as functions of $\Delta \alpha_{R}, \Delta \alpha_{M}$ and $\Delta \alpha_{A}$. Figure 8 also shows the bias and width as functions of $\Delta \alpha_{A}$ for three values of $\Delta \alpha_{P}$. Similar data for the bias and width were collected for each of the five remaining wavelength bands.

An analysis of the trends due to the effects of various atmospheric parameters in measured data determined the requisite accuracy to which each parameter would have to be measured 
to ensure at most $0.1 \% \Phi_{\text {true }}$ contribution to the uncertainty from each $\alpha$ parameter in the fit bias $\left(\sigma_{b i a s, \alpha}\right)$ and at most $1 \% \Phi_{\text {true }}$ uncertainty in the distribution width $\left(\sigma_{\text {width }, \alpha}\right)$. Assuming that the data in Figure 8 follow a linear trend, the slope of each line provides the relationship between the uncertainty in each $\alpha$ parameter and the uncertainty in the bias and width. Table 1 shows the percentage accuracy required in each $\alpha$ parameter for each wavelength band to ensure that $\sigma_{\text {bias }, \alpha}<0.1$ and $\sigma_{\text {width }, \alpha}<1$. Such accuracy in characterizing atmospheric fluctuations reduces the total uncertainty on the corrected flux data.

\section{DISCUSSION AND CONCLUSIONS}

Variations in the Rayleigh scattering parameter, $\alpha_{R}$, produce the largest variations in corrected flux measurements, especially in the lowest wavelength bands, U and G. Figure 8 demonstrates that a $20 \%$ increase in $\alpha_{R}$ changes the fit bias by almost 0.3 and the width by almost 0.5 . The results in Table 1 show that the Rayleigh scattering parameter must be measured to within at most $8 \%$ in the lowest wavelength band. It is known that the Rayleigh scattering extinction coefficient, which depends on the volume density of small particles (radius « incident wavelength), varies mostly with pressure. This volume density can be accurately measured, because $\alpha_{R}$ does not vary by more than $1-2 \%$ throughout the year[6].

Variations in the molecular absorption parameter, $\alpha_{M}$, produce significant variations in the data only in the regions with absorption lines that appear in the three highest wavelength bands (I, Z and Y) for the atmospheric model used in this study. According to Table 1, $\alpha_{M}$ must be measured to within at most $62 \%$ uncertainty. However, this model takes into account only changes in the water content of the atmosphere and assumes that the volume density of other molecules, such as ozone and various oxides, remains constant over time and space. Ozone, sulfur dioxide, ammonia and other variable density molecules constitute about one 
tenth the volume of water molecules in the atmosphere [7], thus the effects of these molecules are not as significant as those of water. A more thorough atmospheric model would take into account variations in density of these molecules as well.

The aerosol scattering parameters $\alpha_{A}$ and $\alpha_{P}$ affect the atmospheric extinction distribution almost uniformly across all wavelength bands (Figure 4), but the effects are not significant enough to cause sensitivity to these parameters in the data. According to Table 1 , the uncertainty on both parameters can be $>100 \%$ in all wavelength bands.

The method of analyzing atmospheric effects discussed in this paper was applied to the spectral energy distribution of galaxy Sbc from [8]. Figure 9 shows the difference in residuals between two pairs of adjoining wavelength bands as functions of redshift, using the initial atmospheric parameters for the atmospheric distribution. These differences in wavelength bands are called "colors" and are used to analyze the chemical makeup of galaxies. The colors fluctuate with redshift by up to several percent $\Phi_{\text {true }}$. These fluctuations are significant and must be accounted for in analyzing distant galaxies.

Changes in atmospheric parameters produce a linear trend in the colors. Figure 10 shows two colors as functions of $\Delta \alpha_{R}$ at zero redshift. The slope of the color vs. $\Delta \alpha_{R}$ data is plotted as a function of redshift in Figure 11. Because the slope does not fluctuate by more than $1 \%, \alpha_{R}$ can be determined with similar accuracy for galaxies at each redshift.

The effects of atmospheric extinction vary with space and time due to changes in temperature, pressure and wind patterns that carry particles throughout the area surrounding the telescope, so as the LSST moves across the sky, it must account for these atmospheric changes in real time. The results of this study provide a good understanding of how the various sources of atmospheric extinction compound the uncertainty on flux data collected by the LSST, and may aid the design of the atmospheric monitoring systems for the telescope. The proposed model [7] is an auxiliary telescope for measuring variation in standard stars due to atmospheric extinction. These standard stars will be used to calibrate measurements taken by the LSST using the method implemented in this study. Ideally, calibrated flux 
measurements collected by the LSST should be within $1 \%$ of the true flux, thus requiring the fit bias and the width about the true flux to be less than $1 \% \Phi_{\text {true }}$. Bias and width values of $>1 \% \Phi_{\text {true }}$, such as those in Figure 8, are due to the intrinsic spread in the data, so reducing these values to less than $1 \% \Phi_{\text {true }}$ requires a deeper understanding of the structure of the spectral energy distributions.

\section{ACKNOWLEDGMENTS}

First and foremost, I would like to thank my mentor, David Burke, for his guidance. I would also like to thank the program directors and Mike Woods for giving me the opportunity to work at SLAC on a project that was entirely new to me. Finally, I would like to thank the Office of Science and the Department of Energy for funding this internship.

\section{REFERENCES}

[1] K. Gilmore, "LSST filter data," 2005.

[2] E. Hubble, "A relation between distance and radial velocity among extra-galactic nebulae," Proc. N. A. S., vol. 15, no. 3, pp. 168-173, 1929.

[3] A. G. Riess et al., "Observational evidence from supernovae for an accelerating universe and a cosmological constant," Astron. J., vol. 116, pp. 1009-1038, 1998.

[4] S. Perlmutter et al., "Measurements of omega and lambda from 42 high-redshift supernovae," Astrophys. J., vol. 517, pp. 565-586, 1999.

[5] J. E. Gunn and L. Stryker, "Stellar spectrophotometric atlas, $3130<\lambda<$ 10800 Å," Astrophys. J. Suppl., vol. 52, pp. 121-153, 1983. [Online]. Available: http://vizier.cfa.harvard.edu/viz-bin/VizieR?-source=III/88 
[6] A. Gutiérrez-Moreno, H. Moreno, and G. Cortés, "A study of the atmospheric extinction at Cerro Tololo inter-american observatory," Publ. Astron. Soc. Pac., vol. 94, pp. 722$728,1982$.

[7] D. Burke and Z. Ivezic, "LSST memorandum: Auxiliary spectroscopic telescope," 2006.

[8] G. D. Coleman, C.-C. Wu, and D. W. Weedman, "Colors and magnitudes predicted for high redshift galaxies," Astrophys. J. Suppl., vol. 43, pp. 393-416, 1980. 


\section{TABLES}

Table 1: Maximum percent uncertainty allowed for each $\alpha$ parameter in (5) so that the uncertainty due to each $\alpha$ on the bias is $\sigma_{\text {bias }, \alpha}<0.1 \% \Phi_{\text {true }}$ and the uncertainty on the width is $\sigma_{\text {width } \alpha}<1 \% \Phi_{\text {true }}$. Such accuracy in the measurement of atmospheric parameters ensures minimal contribution to the uncertainty on the data due to atmospheric fluctuations.

\begin{tabular}{c|cccc|cccc}
\hline \hline & \multicolumn{7}{|c}{ Maximum allowed uncertainty } \\
\cline { 2 - 9 } Filter & $\% \alpha_{R}$ & $\% \alpha_{M}$ & $\% \alpha_{A}$ & $\% \alpha_{P}$ & $\% \alpha_{R}$ & $\% \alpha_{M}$ & $\% \alpha_{A}$ & $\% \alpha_{P}$ \\
\hline $\mathrm{U}$ & 9.19 & $\gg 100$ & 295 & 394 & 39.6 & $\gg 100$ & $\gg 100$ & $\gg 100$ \\
$\mathrm{G}$ & 7.77 & $\gg 100$ & 122 & 230 & 50.4 & $\gg 100$ & 793 & $\gg 100$ \\
$\mathrm{R}$ & 67.1 & $\gg 100$ & 498 & $\gg 100$ & 343 & $\gg 100$ & $\gg 100$ & $\gg 100$ \\
$\mathrm{I}$ & 420 & 537 & $\gg 100$ & $\gg 100$ & $\gg 100$ & $\gg 100$ & $\gg 100$ & $\gg 100$ \\
$\mathrm{Z}$ & $\gg 100$ & 81.5 & $\gg 100$ & $\gg 100$ & $\gg 100$ & 333 & $\gg 100$ & $\gg 100$ \\
$\mathrm{Y}$ & $\gg 100$ & 62.3 & $\gg 100$ & $\gg 100$ & $\gg 100$ & 366 & $\gg 100$ & $\gg 100$ \\
\hline \hline
\end{tabular}




\section{FIGURES}

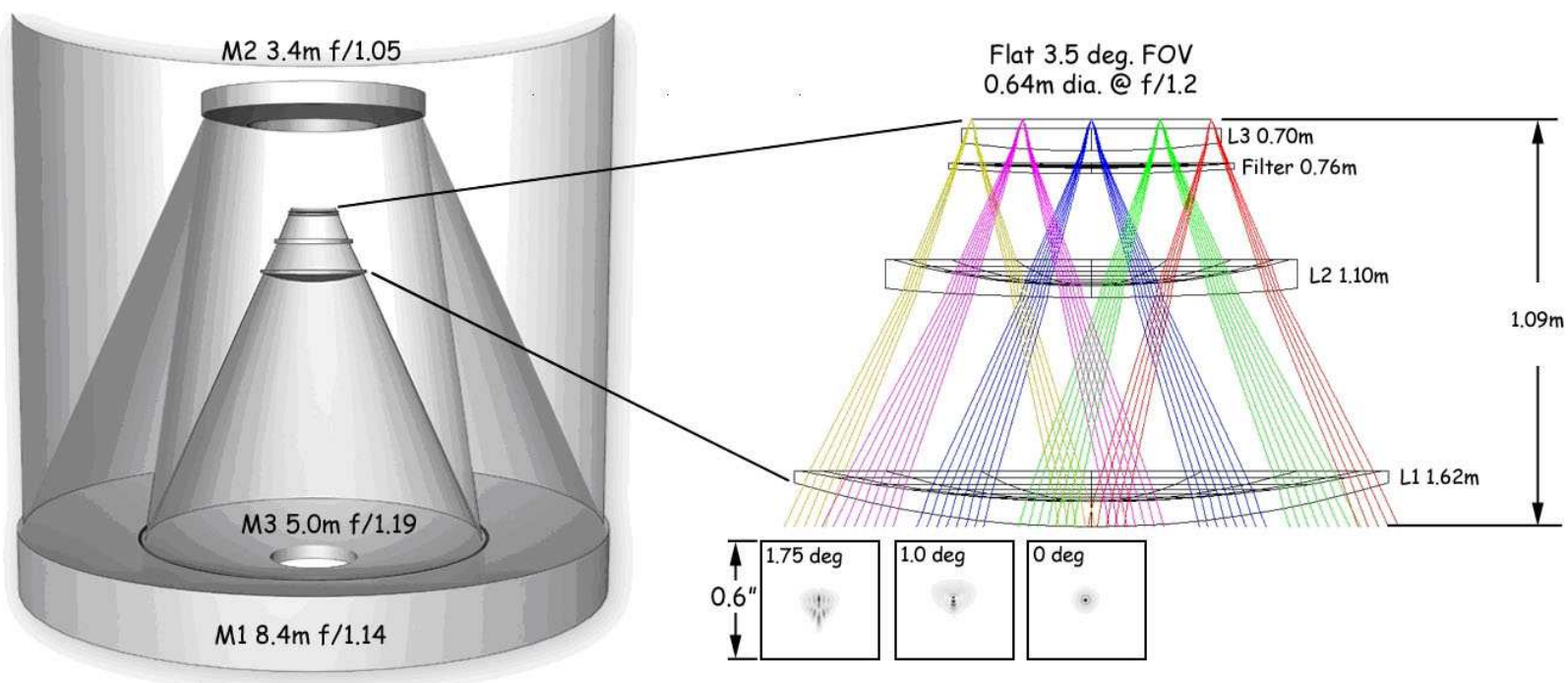

Figure 1: Optical layout of the LSST. The filters are placed $6 \mathrm{~cm}$ from lens 3 (L3), as shown, and select for light in six different wavelength bands. Wavefronts entering the telescope are distorted by the optics and atmosphere through which the light travels. Thus, telescope images are distorted into point spread functions (lower right) with a typical FWHM of $0.6^{\prime \prime}$ due to atmospheric distortion. These images demonstrate that the uncertainty due to atmospheric effects must be reduced in order to reduce uncertainty in the data, since optical effects are much less significant (Credit: LSST Corporation). 


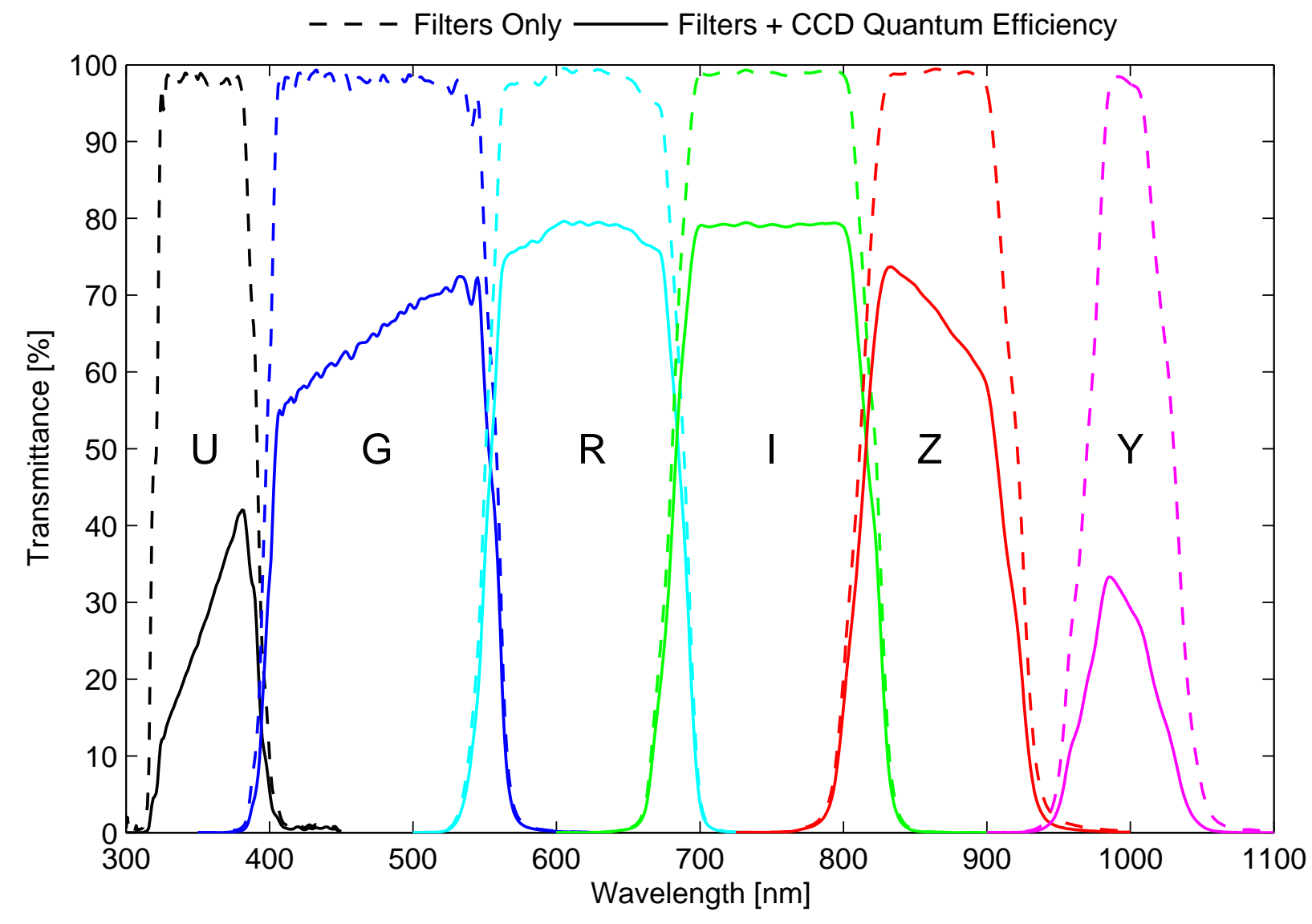

Figure 2: Response functions for each of the six proposed filter bands for the LSST (dashed lines), and response functions that take into account the quantum efficiency of the CCD camera (solid lines). 


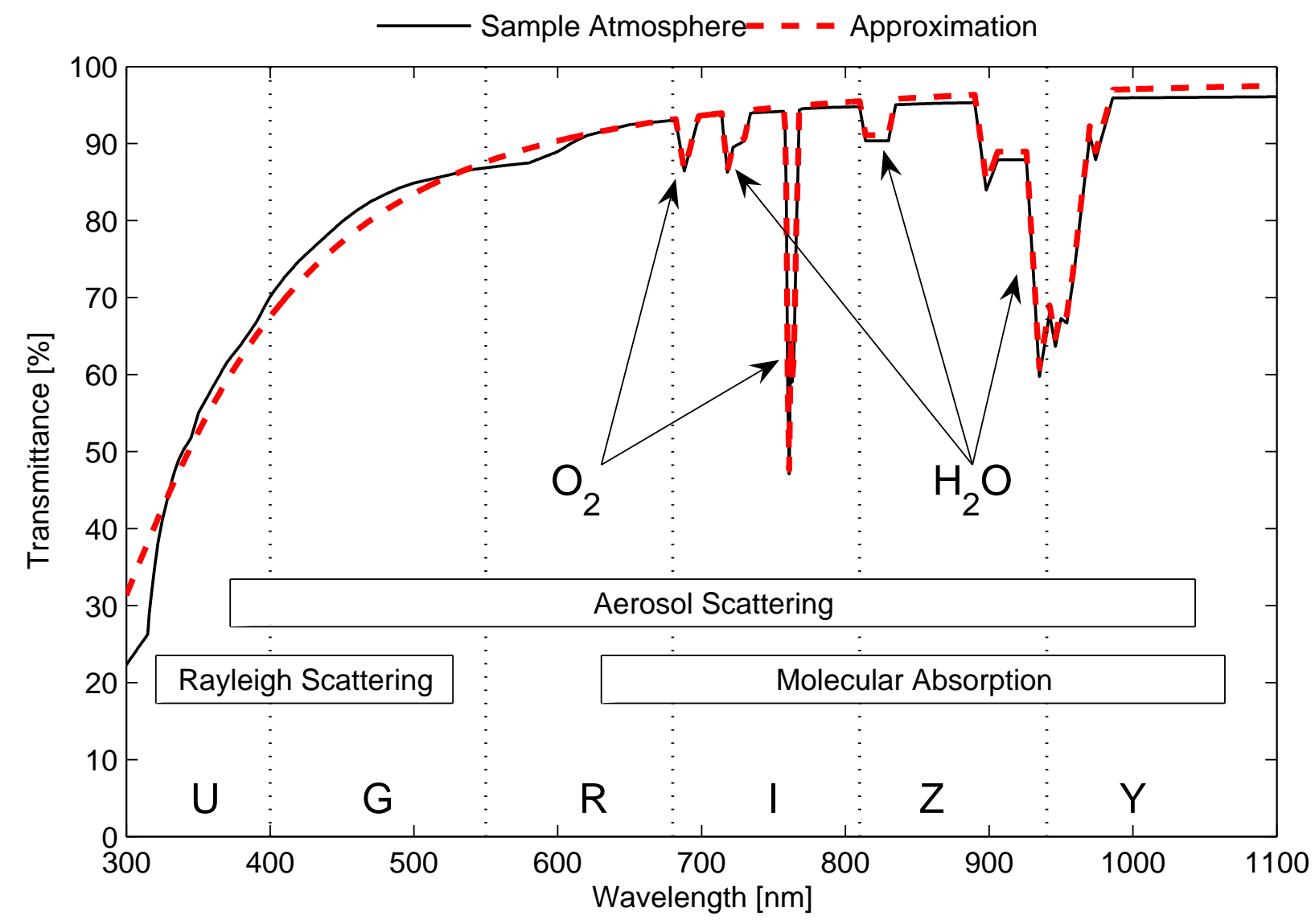

Figure 3: Typical extinction distribution of light entering the atmosphere as a function of wavelength, from [1]. The absorption lines for oxygen and water are illustrated. The range of wavelengths where each extinction process is most significant is also shown. This particular distribution was approximated using (5) with values $\alpha_{R, 0}=1.089, \alpha_{M, 0}=1, \alpha_{A, 0}=0.071$ and $\alpha_{P}=1$. 


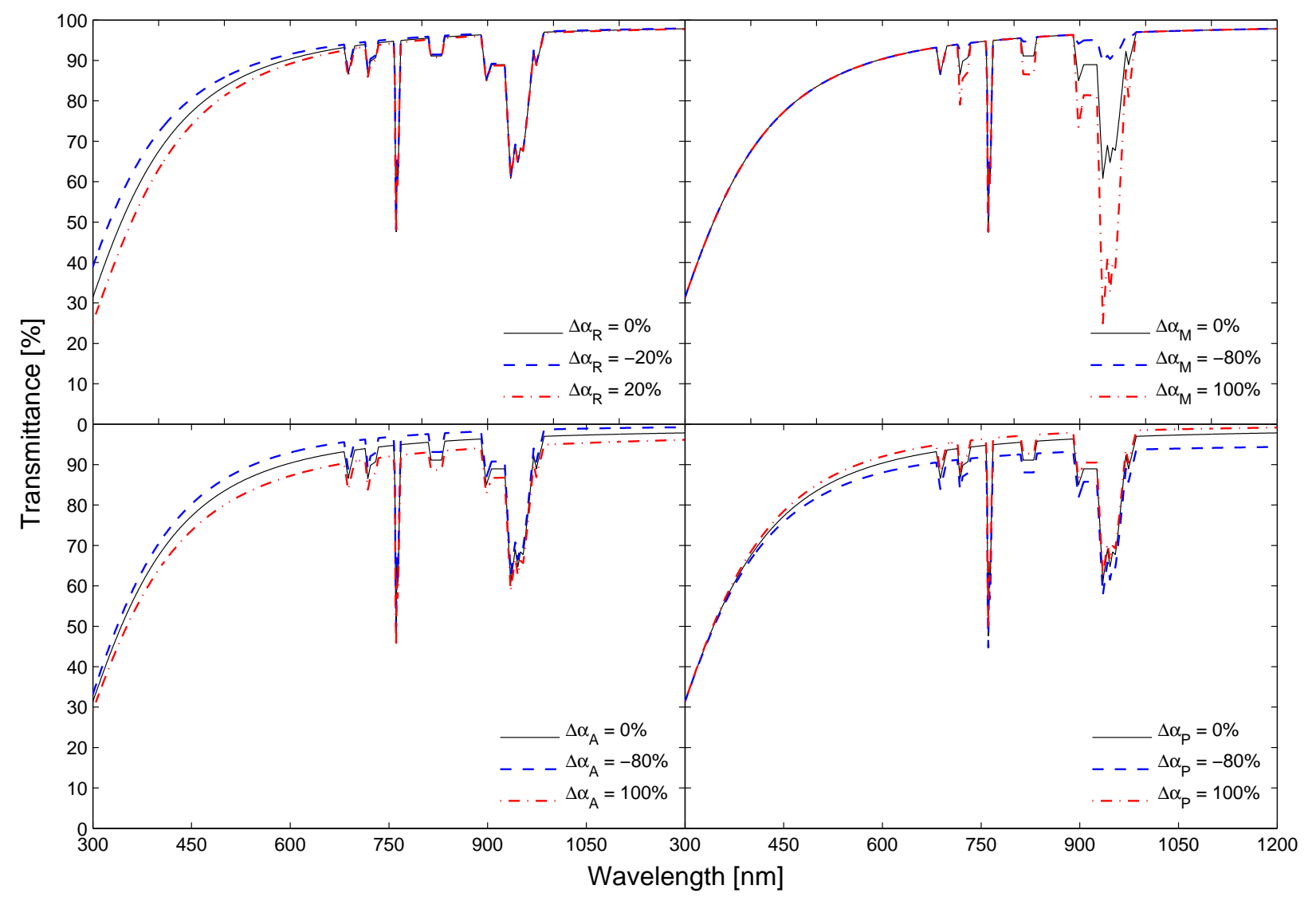

Figure 4: Atmospheric distributions for high and low values of each $\alpha$ parameter. 


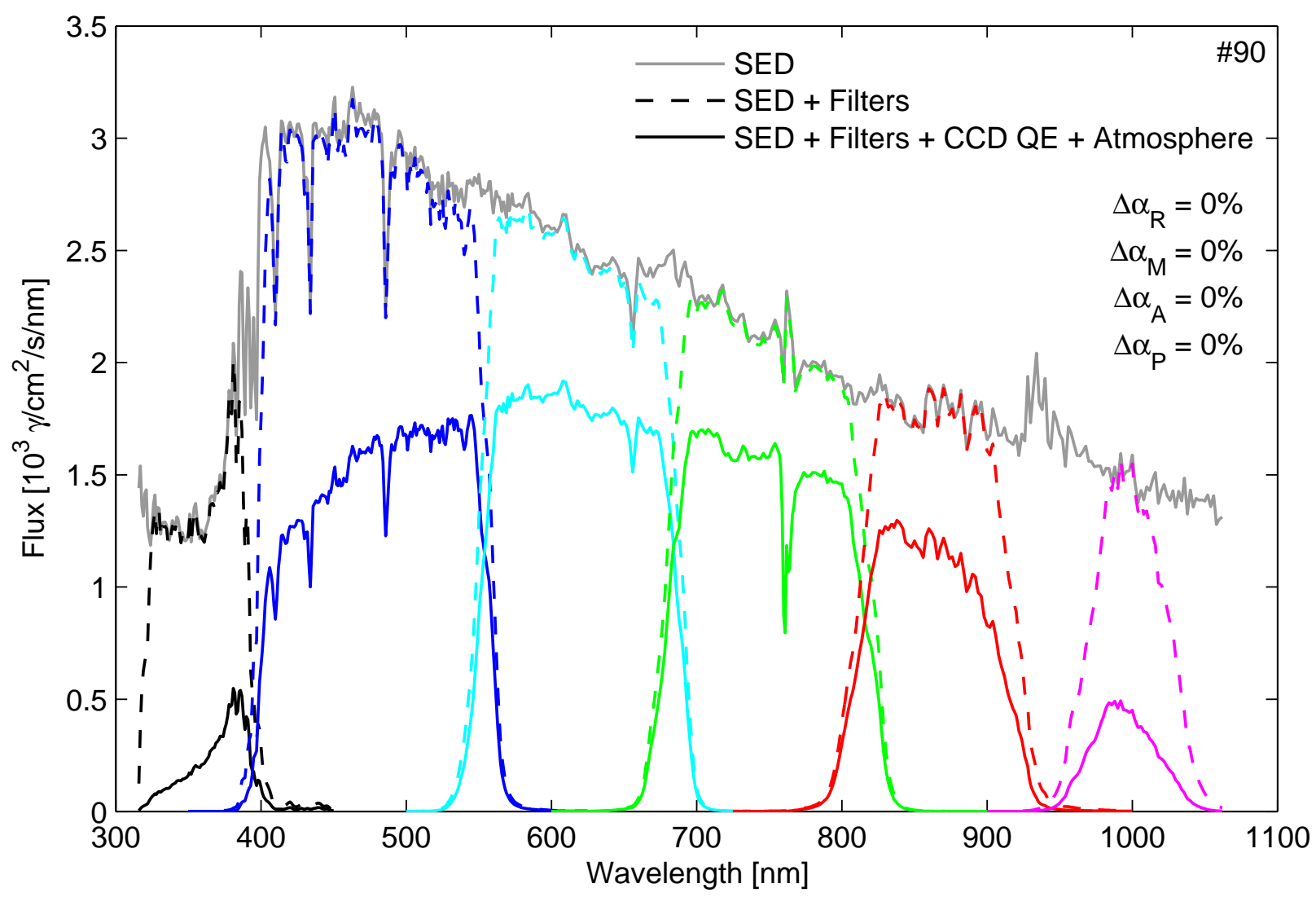

Figure 5: The spectral energy distribution of a giant star (\#90 in [5]). The dashed colored lines show the flux distributions in each filter band. The solid colored distributions include the quantum efficiency of the CCD camera, as well as the atmospheric effects. The integrals of the dashed and solid distributions across each filter band correspond to (9) and (10), respectively. The measured flux is calculated using the atmospheric extinction distribution in Figure 3. 


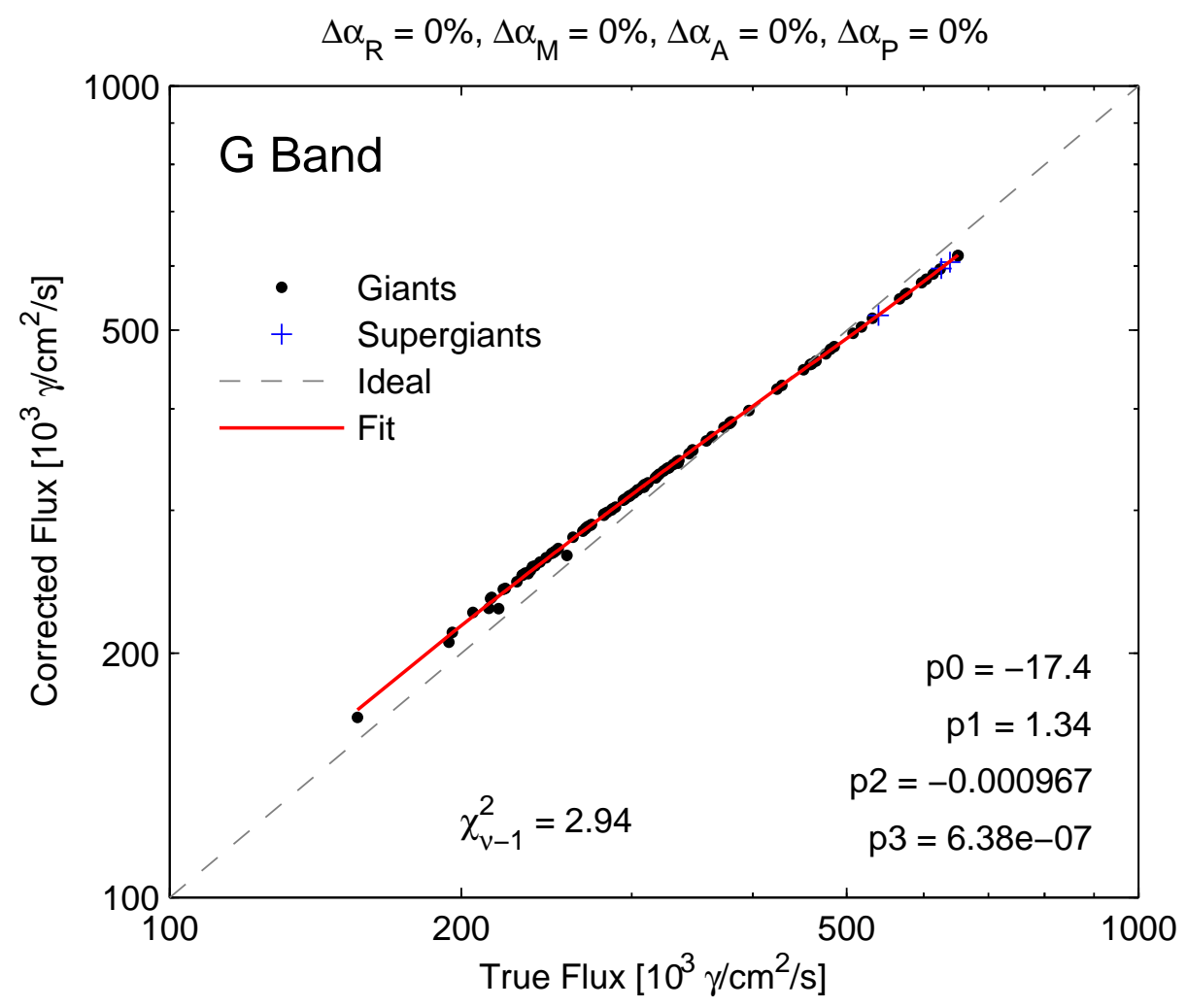

Figure 6: The distribution of corrected flux values in the $G$ band for each of the giant and supergiant stars in [5], fit to a third-order polynomial. The bias of the fit from the ideal line $\left(\Phi_{\text {corr }}=\Phi_{\text {true }}\right)$ and the width of the distribution about the ideal line were calculated for various changes in the atmospheric parameters. 


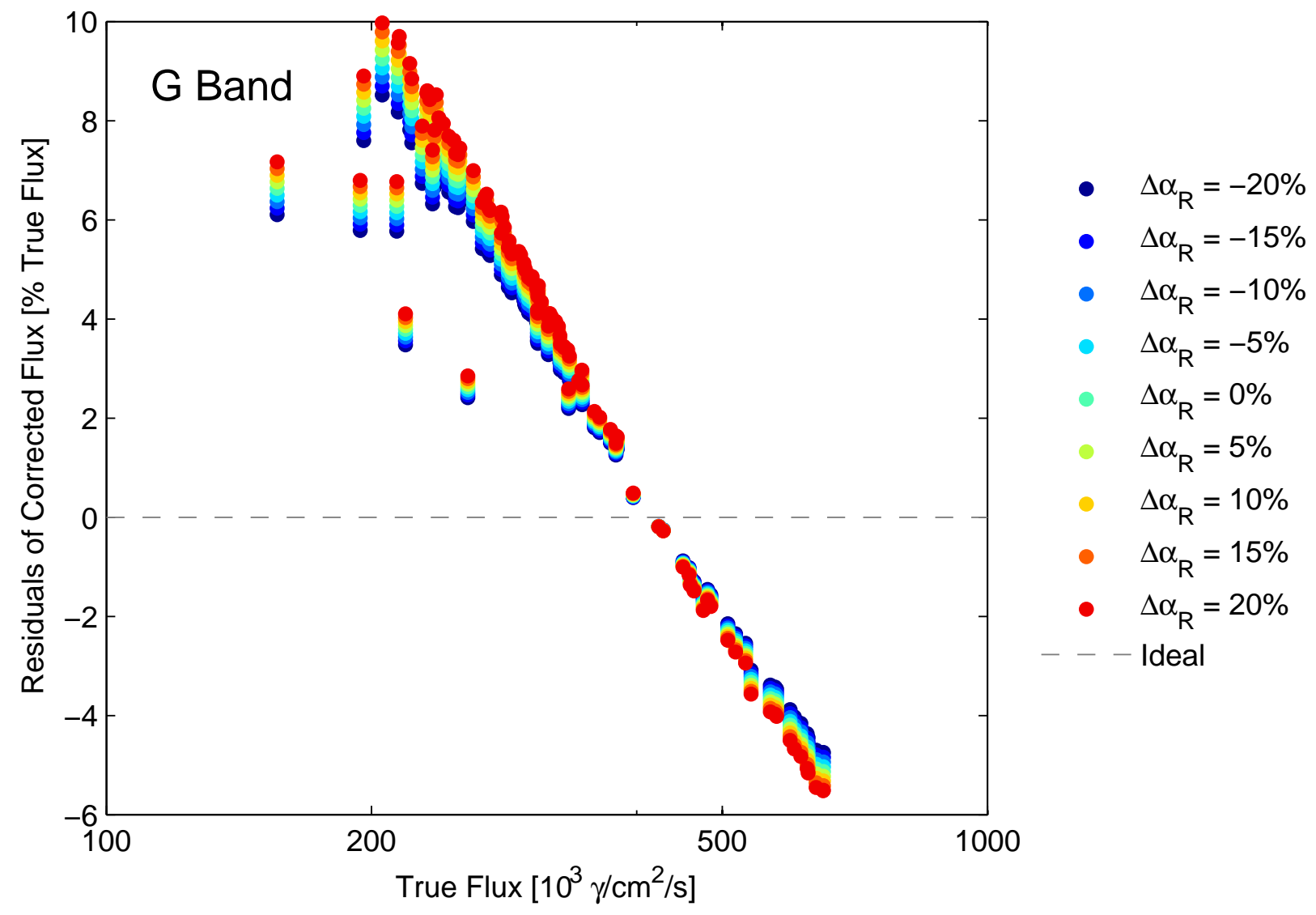

Figure 7: Distribution of residuals $\left(\Phi_{\text {corr }}-\Phi_{\text {true }}\right)$ across the G band for various values of the Rayleigh scattering parameter $\alpha_{R}$, while $\alpha_{M}, \alpha_{A}$ and $\alpha_{P}$ remain fixed to their initial values. The bias of each fit from the ideal line $\left(\Phi_{c o r r}=\Phi_{\text {true }}\right)$ and the width of each distribution about the ideal line were calculated for each of the distributions shown above. 


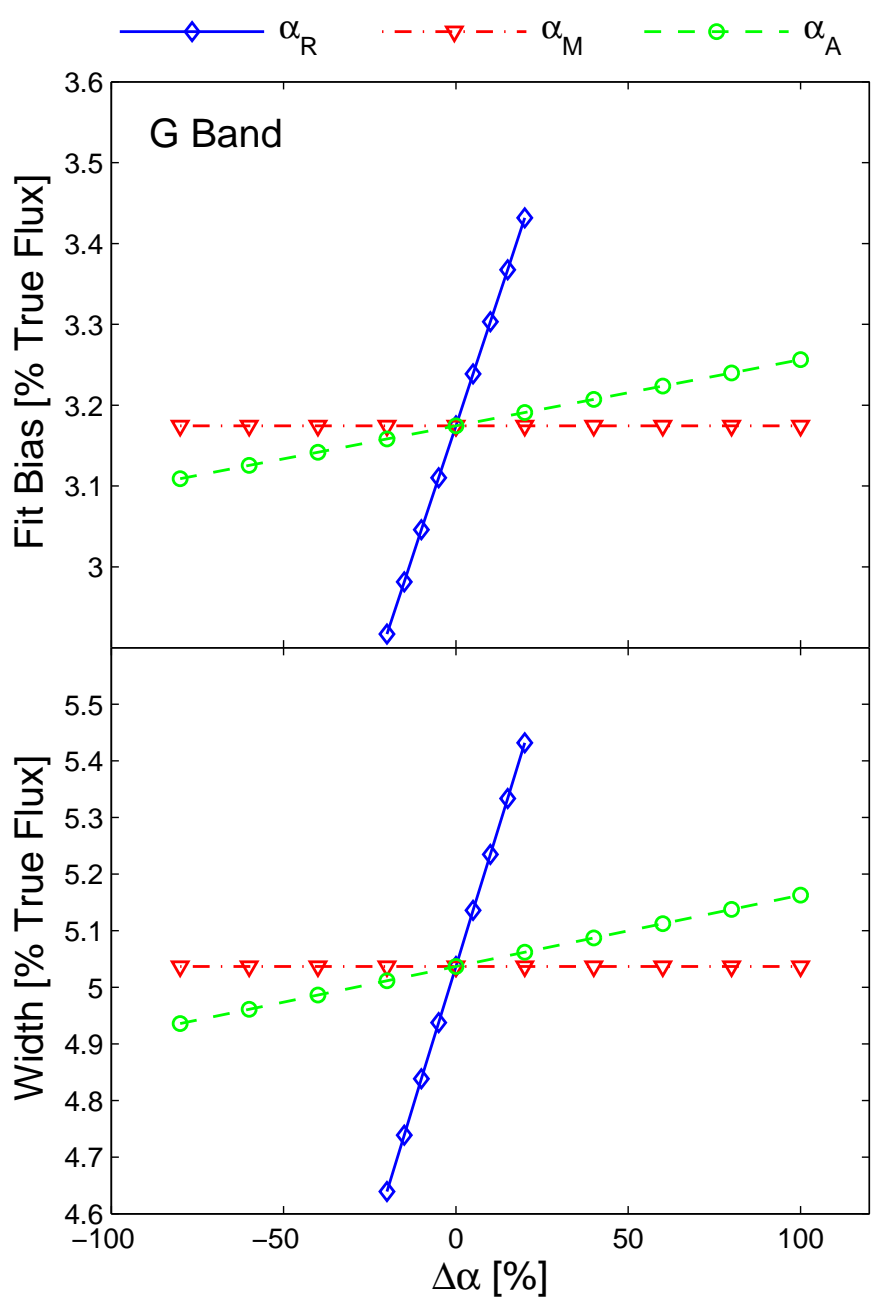

(a) Fit bias and width about the ideal line as functions of $\Delta \alpha_{R}, \Delta \alpha_{M}$ and $\Delta \alpha_{A}$ in the $\mathrm{G}$ band.

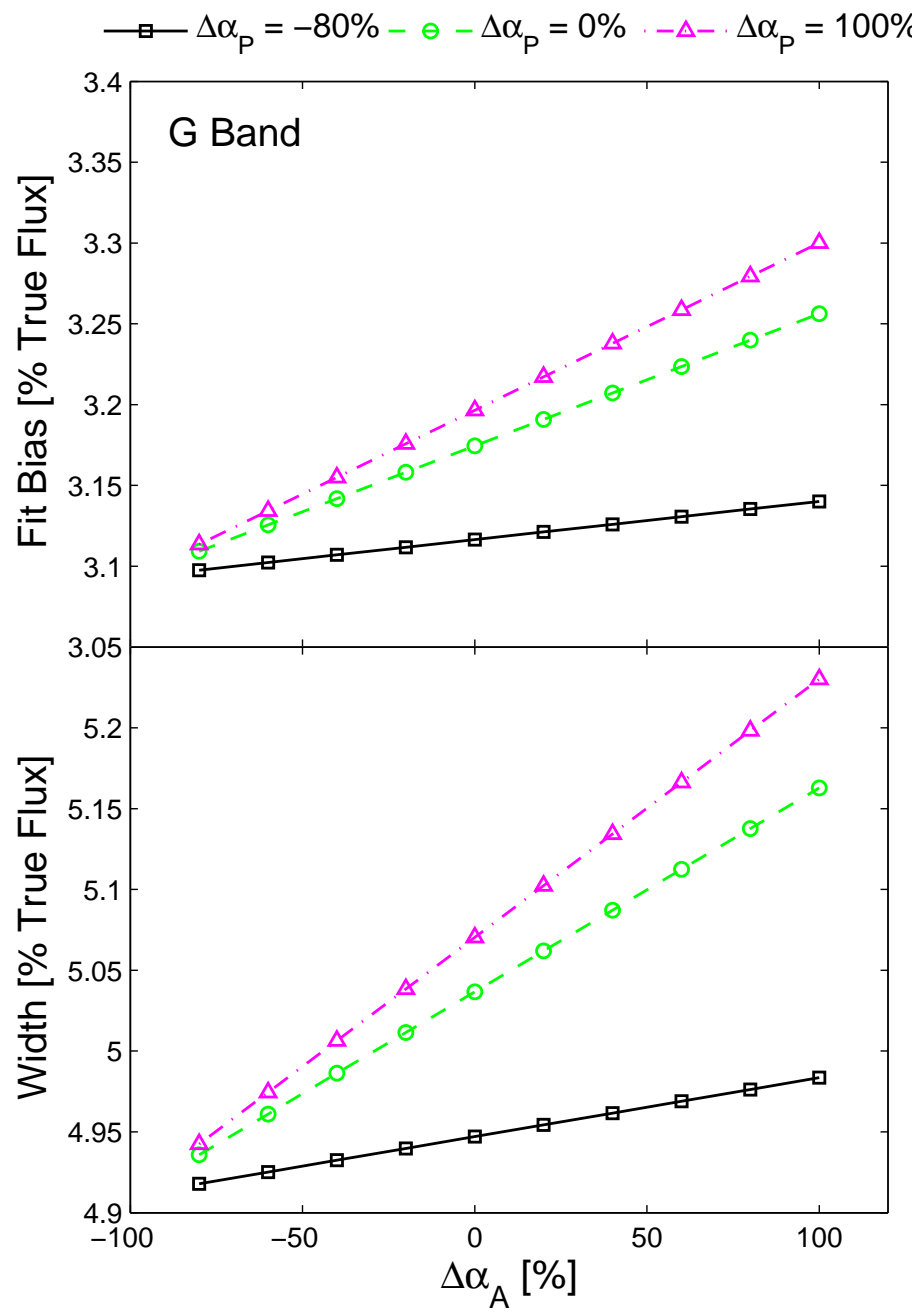

(b) Fit bias and width about the ideal line as functions of $\Delta \alpha_{A}$ for three values of $\Delta \alpha_{P}$ in the $\mathrm{G}$ band.

Figure 8: Small variations in the bias and width imply a lower constraint on the corresponding $\alpha$ parameter, such that the uncertainty in the bias and width is within $0.1 \%$ of the true flux. Larger variations in the bias and width imply a tighter constraint on the $\alpha$ parameter. The variations in trends for each value of $\Delta \alpha_{P}$ in (b) show that the flux values vary as the aerosol content of the atmosphere changes in particle size and density. 


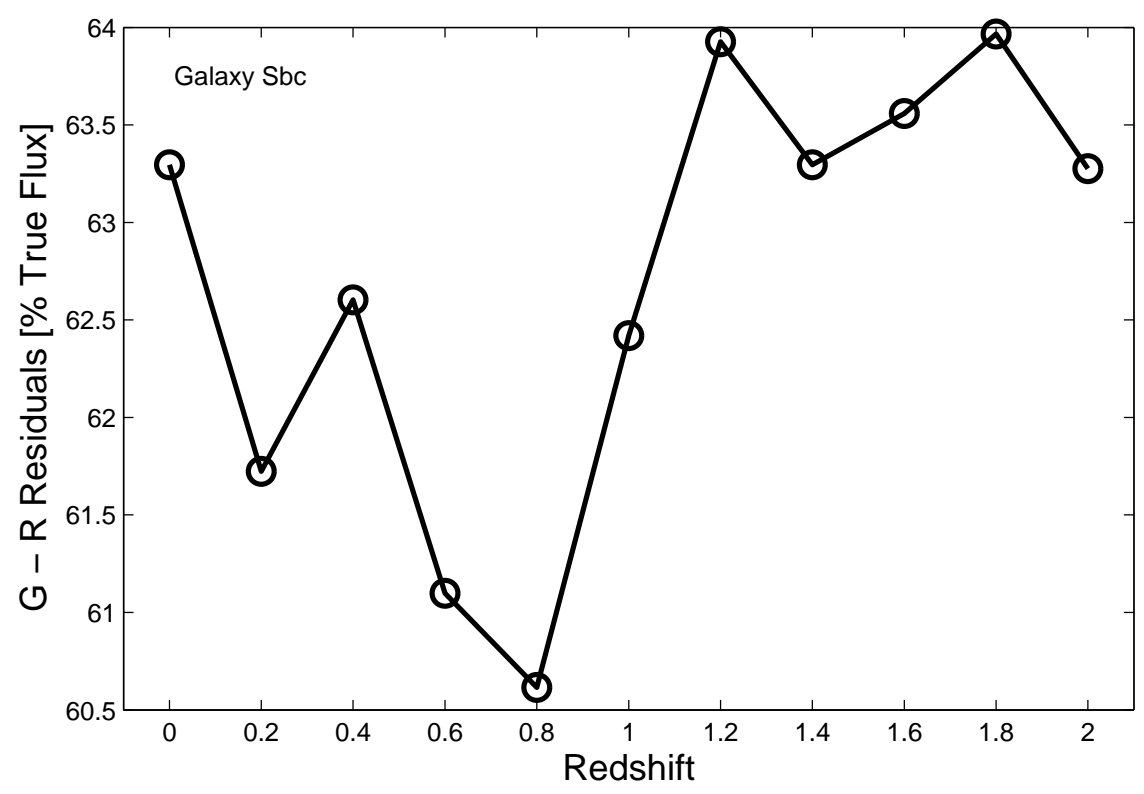

(a)

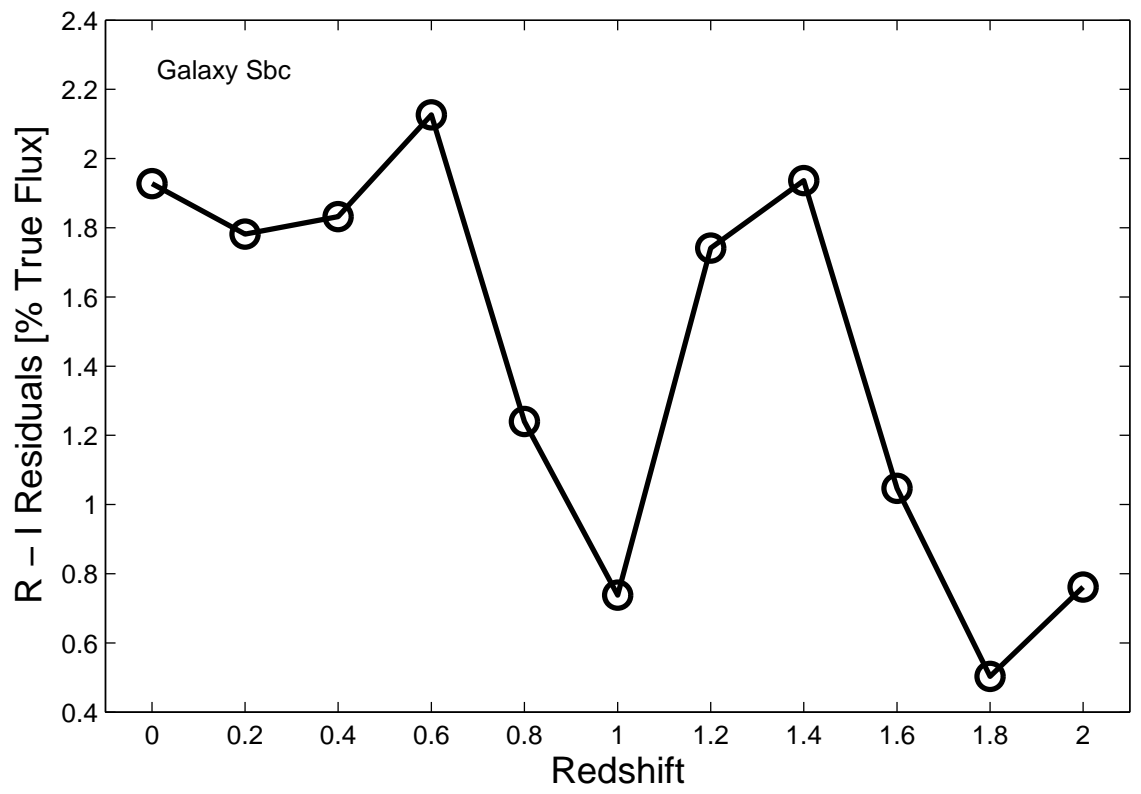

(b)

Figure 9: Residuals of colors $\mathrm{G}-\mathrm{R}$ (a) and R-I (b) as functions of redshift for galaxy Sbc in $[8]$. 


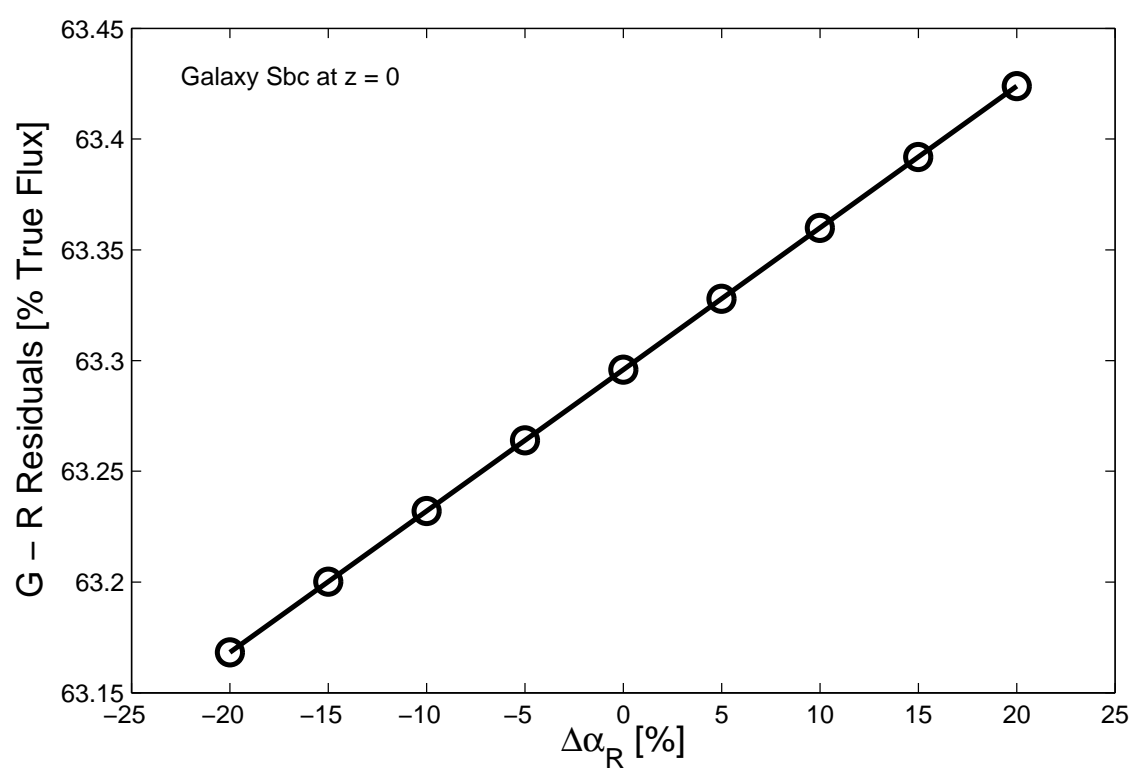

(a)

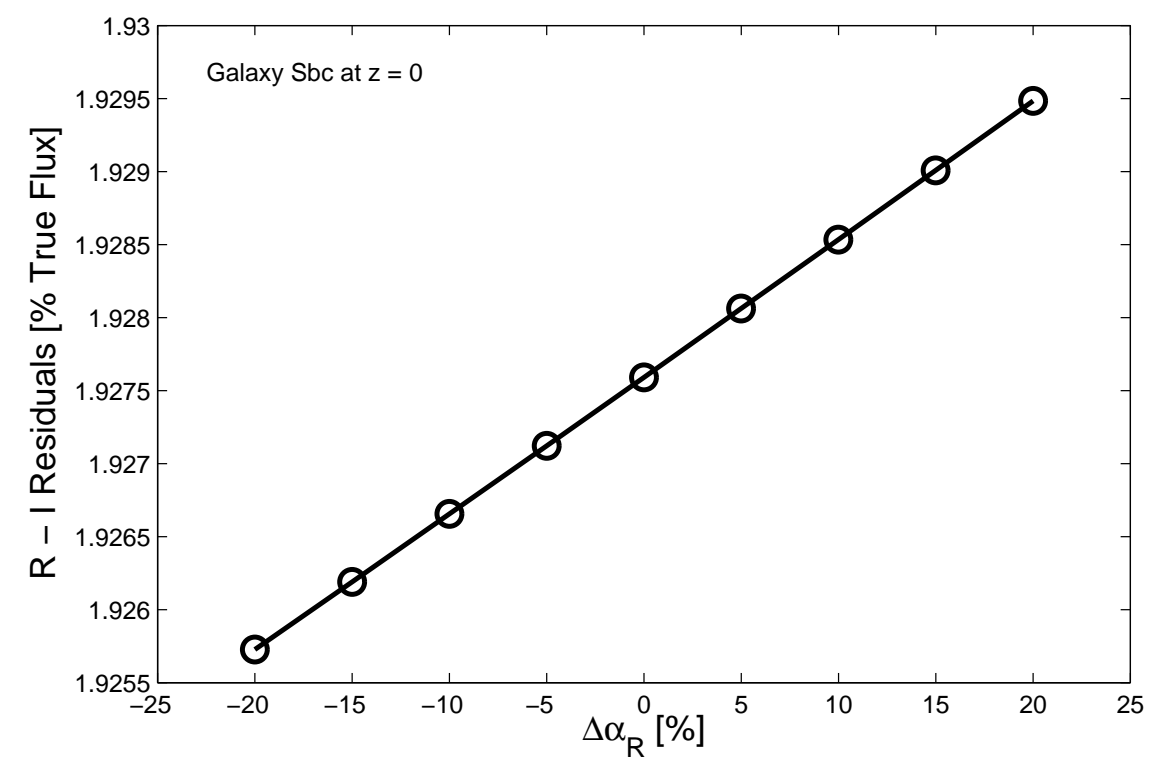

(b)

Figure 10: Residuals of colors $\mathrm{G}-\mathrm{R}$ (a) and R-I (b) as functions of $\Delta \alpha_{R}$ for galaxy Sbc in [8]. 


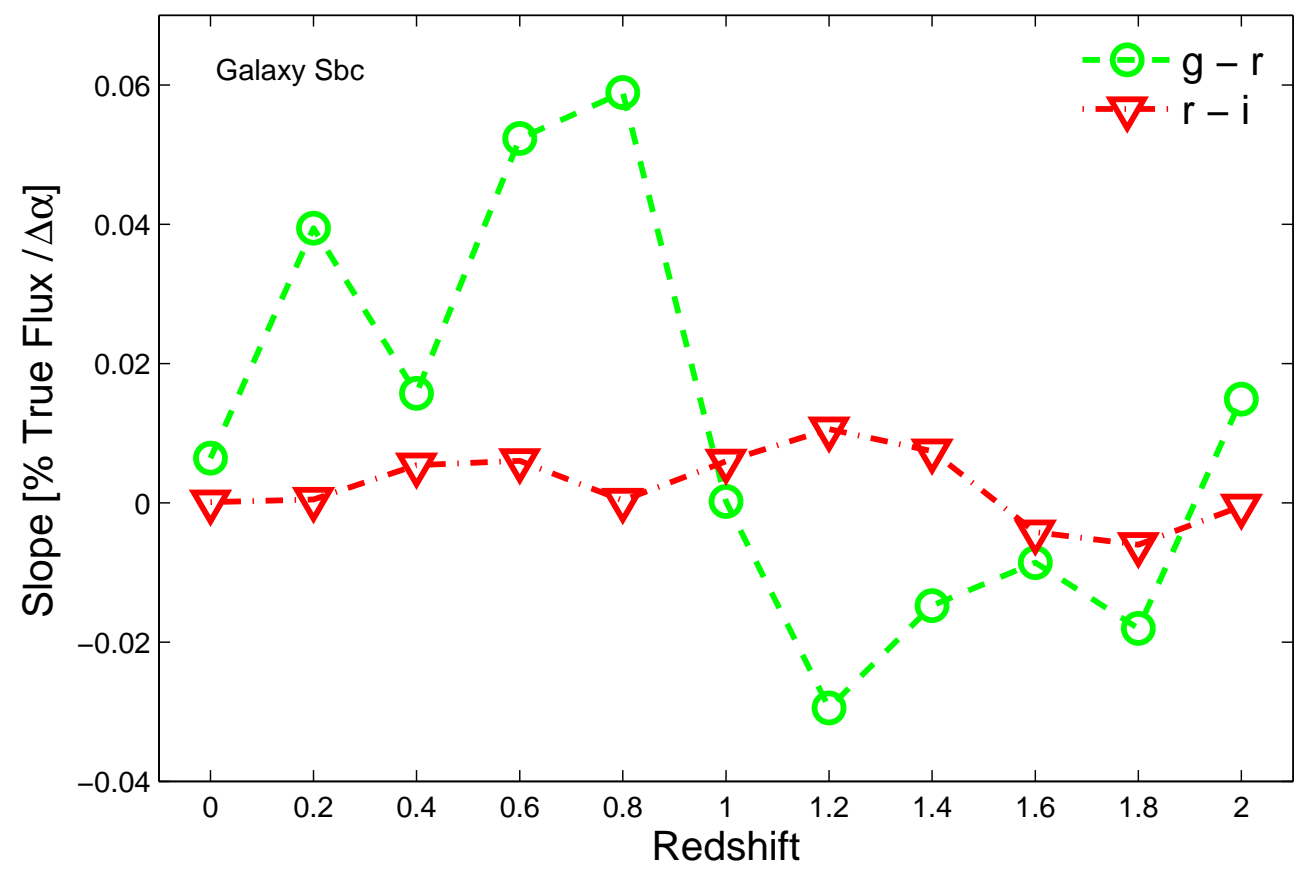

Figure 11: Slope of data in Figure 10 as a function of redshift. 